\title{
Three-Dimensional Laminin Mimetic Peptide Nanofiber Gels for In Vitro Neural Differentiation
}

\author{
Gokhan Gunay, Melike Sever, Ayse B. Tekinay,* and Mustafa O. Guler*
}

The extracellular matrix (ECM) provides biochemical signals and structural support for cells, and its functional imitation is a fundamental aspect of biomaterial design for regenerative medicine applications. The stimulation of neural differentiation by a laminin protein-derived epitope in two-dimensional (2D) and three-dimensional (3D) environments is investigated. The 3D gel system is found to be superior to its $2 D$ counterpart for the induction of neural differentiation, even in the absence of a crucial biological inducer in nerve growth factor (NGF). In addition, cells cultured in 3D gels exhibits a spherical morphology that is consistent with their form under in vivo conditions. Overall, the present study underlines the impact of bioactivity, dimension, and NGF addition, as well as the cooperative effects thereof, on the neural differentiation of PC-12 cells. These results underline the significance of 3D culture systems in the development of scaffolds that closely replicate in vivo environments for the formation of cellular organoid models in vitro.

\section{Introduction}

The extracellular matrix (ECM) provides biochemical cues and structural support for cells, and its functional imitation, despite posing a substantial challenge from a technical standpoint, offers unprecedented levels of control over the molecular processes that drive cellular behaviors such as proliferation and differentiation. The design of biomaterials that emulate the native environment of cells is also crucial for regenerative medicine, as cellular and molecular responses can change significantly depending on the biological, chemical, and physical properties of the immediate environment. Although cells are

G. Gunay, Prof. A. B. Tekinay

Neuroscience Graduate Program

Bilkent University, Ankara, Turkey

E-mail: atekinay@bilkent.edu.tr

M. Sever, Prof. A. B. Tekinay, Prof. M. O. Guler

Institute of Materials Science and Nanotechnology, National

Nanotechnology Research Center (UNAM)

Bilkent University, Ankara, Turkey

Prof. M. O. Guler

Institute for Molecular Engineering

University of Chicago, Chicago, IL 60637, USA

E-mail:mguler@uchicago.edu

The ORCID identification number(s) for the author(s) of this article can be found under https://doi.org/10.1002/biot.201700080.

DOI: $10.1002 /$ biot.201700080 widely cultured in two-dimensional (2D) environments, ${ }^{[1]}$ recent studies have indicated that cellular morphology, viability, ${ }^{[2,3]}$ proliferation, ${ }^{[4]}$ signaling, ${ }^{[5]}$ and differentiation $^{[6,7]}$ are more similar to in vivo conditions in three-dimensional (3D) systems. The 3D hydrogels such as Matrigel ${ }^{\mathbb{R}}$, collagen and alginate are extensively used in cell culture studies, and their similarity to the natural extracellular matrix contributes strongly to their bioactivity in regenerative medicine applications. ${ }^{[8]}$ The $3 \mathrm{D}$ collagen gels, for example, are highly suitable for mimicking the connective tissue,$^{[9]}$ while Matrige ${ }^{\mathbb{R}}$ is applicable for the culture of a broad range of cells and tissues due to its incorporation of basement membrane elements such as laminin, collagen IV, and entactin. ${ }^{[10]}$ However, these materials present significant batchto-batch variation and do not necessarily provide the full range of ECM-mimetic signals found in natural tissue environments. ${ }^{[11,12]}$ Consequently, there is considerable potential in developing easy-to-use and tailorable 3D culture systems through the use of synthetic biomaterials.

Self-assembling peptide amphiphile nanofiber gels have been widely used in $2 \mathrm{D}$ and $3 \mathrm{D}$ cellular culture studies ${ }^{[13]}$ and are promising candidates for regenerative medicine applications due to their biocompatibility, biodegradability, and incorporation of bioactive signals. ${ }^{[14,15]}$ Peptide amphiphile systems have been extensively used in neural regeneration applications, and bioactive epitopes derived from ECM proteins, such as the laminin-derived IKVAV and YIGSR, fibronectin-derived RGD, and tenascin-C-derived VFDNFVLK sequences, have been shown to induce neural differentiation, attachment, and migration. ${ }^{[16-19]}$ The imitation of ECM proteins is especially important in this context, as integrin receptors and similar mechanotransduction elements are essential for cellular adhesion, homing, and differentiation. ${ }^{[20]}$ Many types of integrins, in turn, are directly bound to ECM components ${ }^{[21]}$ and initiate signaling cascades through focal adhesion kinase (FAK) and phosphoinositide 3-kinase pathways. $\beta 1$ integrins, for example, were shown to be effective for stem cell differentiation and selfrenewal. ${ }^{[22]}$ Previous studies also indicated that $\beta 1$ integrins regulate the self-renewal and differentiation of neural stem cells (NSCs) through Notch and EGF receptors. ${ }^{[23]}$ Laminin is another major ECM component of both central and peripheral nervous systems (CNS and PNS) and serves as an important mediator of axonal outgrowth. ${ }^{[24]}$ IKVAV is one of the most extensively 
studied bioactive epitopes and is located on the $\alpha$-laminin chain, ${ }^{[25]}$ which is essential for the ability of laminin to regulate the overall cellular fate. ${ }^{[26]}$ The IKVAV peptide sequence, derived from laminin, was previously shown to induce neural differentiation, ${ }^{[27]}$ axonal growth after spinal cord injury in vivo $^{[28]}$ and neural differentiation of PC-12 cells in vitro ${ }^{[17]}$ as a part of peptide nanofiber system. However, no 3D study has so far demonstrated the effect of a laminin-mimetic environment on neuronal differentiation, which is a critical step for the translation of biomimetic scaffolds to clinical applications.

Cell behavior is influenced strongly by the interactions that occur at the cell-biomaterial interfaces, and the development of novel biomaterials that can exercise precise control over cell function are of fundamental importance for tissue regeneration. Consequently, physical properties should also be taken into account in addition to biological and chemical properties when designing biomaterials for tissue-engineering applications. Parameters such as stiffness and dimensionality are important for scaffold functionalization to induce neural differentiation. When designing a scaffold for neural differentiation, the mechanical properties of the scaffold should be similar to that of brain tissue, the elastic modulus of which is below $1 \mathrm{kPa} .^{[29]}$ Stiffness is also important for differentiation into different cell types. For instance, NSCs prefer to differentiate into neurons under an intermediate substrate stiffness (500 Pa), while increased stiffness favors differentiation into the astrocyte lineage. ${ }^{[30]}$ Dimensionality is another important parameter, and although two-dimensional (2D) cell cultures are commonly used in differentiation studies, three-dimensional cell cultures are important in vitro models to fill the gap between 2D cell culture experiments and in vivo studies. 3D models are especially important for studying the regeneration of neural tissue, which has a very low regeneration capacity that may be improved by closely mimicking the native extracellular matrix of neural cells to provide support and enhance the diffusion of oxygen and nutrients. ${ }^{[31]}$

Here, the neural differentiation potential of PC-12 cells is investigated on 2D and 3D laminin-mimetic environments produced through the self-assembly of peptide nanofibers. Effects of bioactivity, dimensionality, and NGF addition on neural differentiation were analyzed by monitoring the expression of neurogenic markers and proteins, and scanning electron microscopy imaging was used to observe the overall morphology of cells in 2D and 3D scaffolds. Overall, the present study compares the neural differentiation potential of PC-12 cells under different culturing conditions and highlights the importance of presenting bioactive IKVAV sequence on 3D hydrogels.

\section{Experimental Section}

\subsection{Materials}

All protected amino acids, lauric acid, 4-(2',4'-dimethoxyphenylFmoc-aminomethyl)-phenoxyacetamido-norleucyl-MBHA resin (Rink amide MBHA resin), 2-(1H-benzotriazol-1-yl)-1,1,3,3tetramethyluroniumhexafluorophosphate (HBTU), and diisopropylethylamine (DIEA) were purchased from Nova-Biochem, ABCR, or Sigma-Aldrich. Cell culture materials were purchased from Invitrogen. All other chemicals and materials used in this study were purchased from Thermo Scientific or Sigma-Aldrich.

\subsection{Peptide Amphiphile (PA) Synthesis and Purification}

PA molecules were synthesized by using an Fmoc solid phase peptide synthesis method on Rink amide MBHA resin. Positively charged LN-PA (lauryl-VVAGKKIKVAV-Am) was used as a bioactive laminin-mimetic PA molecule. Positively charged KK-PA (lauryl-VVAGKK-Am) and negatively charged EE-PA (lauryl-VVAGEE-Am) were used as nonbioactive molecules for charge neutralization and as epitope-free controls. Couplings of amino acids were done by mixing 2 equivalents of amino acids with 1.95 equivalents of HBTU and 3 equivalents of DIEA for $2 \mathrm{~h}$. Fmoc removal was carried out with $20 \%$ piperidine-dimethylformamide (DMF) solution for $20 \mathrm{~min}$. After each coupling step, $10 \%$ acetic anhydride-DMF solution was used to permanently acetylate the unreacted amine groups. After each step, DMF and dichloromethane (DCM) were used as washing solvents. Cleavage of PA molecules and protection groups from the resin was carried out by using a mixture of trifluoroacetic acid (TFA): triisopropylsilane (TIS): $\mathrm{H}_{2} \mathrm{O}$ at a 95:2.5:2.5 ratio for $3 \mathrm{~h}$. Rotary evaporation was used for the removal of excess TFA. Ice-cold diethyl ether was used for the precipitation of PAs in the remaining solution. One day later, centrifugation was used to collect the precipitate, which was dissolved in ultrapure water. The resulting solution was frozen at $-80^{\circ} \mathrm{C}$ for $4 \mathrm{~h}$ and then lyophilized for 3-4 days. Characterization of PAs was carried out by using liquid chromatography-mass spectrometry (LC-MS). Mass spectra were obtained with Agilent LC-MS equipped with Agilent 6530 Q-TOF with an ESI source and Zorbax Extend-C18 $2.1 \times 50 \mathrm{~mm}$ column for basic conditions and a Zorbax SB-C8 $4.6 \times 100 \mathrm{~mm}$ column for acidic conditions. A gradient of (a) water $\left(0.1 \%\right.$ formic acid or $\left.0.1 \% \mathrm{NH}_{4} \mathrm{OH}\right)$ and (b) acetonitrile $\left(0.1 \%\right.$ formic acid or $\left.0.1 \% \mathrm{NH}_{4} \mathrm{OH}\right)$ was used for LC-MS. In order to remove the residual TFA, positively charged PAs were treated with $0.1 \mathrm{M} \mathrm{HCl}$ solution and then lyophilized. Peptide purification was carried out with an Agilent preparative reversephase HPLC system equipped with a Zorbax Extend-C18 $21.2 \times 150 \mathrm{~mm}$ column for basic conditions and a Zorbax SBC8 $21.2 \times 150 \mathrm{~mm}$ column for acidic conditions. A gradient of (a) water $\left(0.1 \%\right.$ TFA or $\left.0.1 \% \mathrm{NH}_{4} \mathrm{OH}\right)$ and (b) acetonitrile $(0.1 \%$ TFA or $0.1 \% \mathrm{NH}_{4} \mathrm{OH}$ ) was used for HPLC. All peptide batches were freeze-dried and dissolved in ultrapure water before use.

\subsection{Scanning Electron Microscopy (SEM)}

SEM was used to visualize the PA nanofiber networks. Thirty milliliters of oppositely charged PA solutions were mixed to produce gel structures through charge neutralization. Gels were prepared on silicon wafers and then dehydrated by using increasing ethanol concentrations of 20, 40, 60, 80, and 100\% v/ v. A Tousimis Autosamdri 815B critical point dryer was used to remove residual ethanol and dried gels were coated with $4 \mathrm{~nm}$ $\mathrm{Au} / \mathrm{Pd}$ prior to imaging. SEM (FEI Quanta 200 FEG), images were taken using an Everhart-Thornley Detector (ETD) system at high vacuum mode at $5 \mathrm{keV}$ beam energy. 


\subsection{Circular Dichroism (CD)}

Oppositely charged PA solutions were mixed at a concentration of $2.5 \times 10^{-4} \mathrm{M}$ in appropriate volumes in order to obtain nanofibers with neutral charges. A total of $120 \mu \mathrm{L}$ of LN-PA and $180 \mu \mathrm{L}$ of EE-PA were mixed to obtain charge neutralization for laminin mimetic peptide nanofibers. A total of $150 \mu \mathrm{L}$ of KK-PA was mixed with $150 \mu \mathrm{L}$ EE-PA for epitope-free control peptide nanofibers with neutral charge (1:1 ratio for KK-PA/EE-PA and 2:3 ratio for LN-PA/EE-PA nanofibers). Measurements were carried out using a Jasco J815 CD spectrometer from 300 to $190 \mathrm{~nm}$ by adjusting data interval and data pitch to $0.1 \mathrm{~nm}$ and scanning speed to $100 \mathrm{~nm} \mathrm{~min}^{-1}$. Digital Integration Time (DIT) was adjusted to $1 \mathrm{~s}$ and band width to $1 \mathrm{~nm}$. All measurements were repeated 3 times.

\subsection{Oscillatory Rheology}

An Anton Paar Physica RM301 Rheometer, operating with a $25 \mathrm{~mm}$ parallel plate configuration at $25^{\circ} \mathrm{C}$, was used for the measurements. Different concentrations of PA molecules were prepared in a total of $250 \mu \mathrm{L}$ and loaded onto the lower plate center, and gels were then incubated for $10 \mathrm{~min}$ before each measurement to ensure complete charge neutralization and gelation. After reaching equilibrium, the upper plate was lowered to have a gap distance of $0.5 \mathrm{~mm}$. Storage modulus $\left(G^{\prime}\right)$ and loss modulus $\left(G^{\prime \prime}\right)$ values were scanned from 100 to $0.1 \mathrm{rad} \mathrm{s}^{-1}$ of angular frequency, with a $0.5 \%$ shear strain. All measurements were repeated 3 times.

\subsection{Zeta Potential}

Zeta potential of the nanofibers was measured by ZetaSizer. A Malvern Nanosizer/ZetaSizer Nano-ZS ZEN 3600 (Malvern Instruments, USA) instrument was used for analysis of charges of the nanofibers. Measurements were performed in quartz cuvettes and repeated three times. Samples were prepared by dissolving each component in water at a concentration of $250 \mu \mathrm{M}$.

\subsection{In Vitro Studies}

PC-12 cells were used in all cell culture experiments. $25 \mathrm{~cm}^{2}$ flasks were used for culturing cells in a humidified incubator at $37^{\circ} \mathrm{C}$, supplied with $5 \% \mathrm{CO}_{2}$. Cells were maintained in Roswell Park Memorial Institute (RPMI) medium supplemented with $10 \%$ horse serum (HS), $5 \%$ fetal bovine serum (FBS), $2 \mathrm{mM} 1$ glutamine, and $1 \%$ penicillin/streptomycin $(\mathrm{P} / \mathrm{S})$. Medium was changed every 3 days.

2D cell culture was carried out by coating positively and negatively charged PA solutions to 6-well plates, which were incubated at $37^{\circ} \mathrm{C}$ for $30 \mathrm{~min}$ and placed in a laminar flow hood at room temperature overnight for the evaporation of solvent. The PA matrix formed on the plates was UV-sterilized for $45 \mathrm{~min}$ prior to cell seeding. PC-12 cells were seeded onto the plates at a density of $1.5 \times 10^{5}$ cells/well. For $3 \mathrm{D}$ gel formation, positively charged PA molecules were dissolved in $30 \mu \mathrm{L}$ of $0.25 \mathrm{M}$ sucrose and negatively charged PA molecules were dissolved in $30 \mu \mathrm{L}$ of culture medium. Initially the first layer of the 3D gel was prepared, and gels were placed into the incubator for stabilization for $1 \mathrm{~h}$. On this gel, positively charged PA solution was added, and negatively charged PA solution containing PC-12 cells at a density of $5 \times 10^{5}$ cells/well was added very slowly in order not to impair gel structure during self-assembly. Gels containing the cells were placed into the incubator for stabilization, and medium was added to each well after $1 \mathrm{~h}$. Cellular differentiation was induced after 1 day with differentiation medium supplemented with $20 \mathrm{ng} \mathrm{mL}{ }^{-1} \mathrm{NGF}$ and the experiment was ended at the end of 7 days (Figure S5, Supporting Information).

\subsection{Neurite Extension Assay}

$2 \mathrm{mM}$ LN-PA and $3 \mathrm{mM}$ EE-PA were used to form gels on 6-well plates and $3 \mathrm{mM}$ KK-PA and $3 \mathrm{mM}$ EE-PA were used as a control group. After coating, plates were incubated at $37^{\circ} \mathrm{C}$ for $30 \mathrm{~min}$, and excess solvent was evaporated in a laminar flow hood at room temperature. Plates were UV-sterilized prior to cell seeding. PC-12 cells were seeded onto the plates at a density of $1.5 \times 10^{5}$ cells/well. Cells were initially seeded in RPMI medium supplemented with $10 \% \mathrm{HS}, 5 \%$ FBS, $2 \mathrm{mM} 1$ glutamine, and $1 \% \mathrm{P} / \mathrm{S}$, and the medium was changed after 1 day with differentiation medium containing MEM with $2 \%$ $\mathrm{HS}, 1 \% \mathrm{FBS}, 2 \mathrm{mM}$ l-glutamine, and $1 \% \mathrm{P} / \mathrm{S}$ along with $20 \mathrm{ng} \mathrm{mL}^{-1} \mathrm{NGF}$. At the end of 7 days, light microscope images were taken at six random points for each well at $200 \times$ magnification. Image J program was used to quantify neurite length and these values were normalized by cell number/image. Neurite-bearing cells were also analyzed by using Image J. Overall results were quantified by counting cells bearing neurite/total cell number (\%). Statistical analysis of the results was performed by one way ANOVA.

\subsection{SEM Imaging of PC-12 Cells Cultured on 2D and in 3D Scaffolds}

SEM (FEI Quanta 200 FEG) imaging was carried out in order to evaluate the morphology and neurite extension pattern of PC-12 cells by using an ETD detector at high vacuum mode at $5 \mathrm{keV}$ beam energy. After 7 days of culturing, cells were washed once with $1 \times$ PBS and fixed with $2 \%$ gluteraldehyde/PBS and $1 \mathrm{wt} \%$ $\mathrm{OsO}_{4}$ for $1 \mathrm{~h}$ each. After fixation, cells were washed with $\mathrm{ddH}_{2} \mathrm{O}$ and dehydrated by using increasing ethanol concentrations of $20,40,60,80$, and $100 \% \mathrm{v} / \mathrm{v}$. A Tousimis Autosamdri 815B critical point dryer was used afterwards and dried samples were coated with $4 \mathrm{~nm} \mathrm{Au} / \mathrm{Pd}$ prior to imaging.

\subsection{Gene Expression Analyses}

Quantitative real time-PCR (qRT-PCR) was used to quantify the gene expression profiles of PC-12 cells cultured on $2 \mathrm{D}$ and in $3 \mathrm{D}$ scaffolds. RNA was isolated from cells at the end of 7 days using TRIzol (Invitrogen) according to the manufacturer's instructions. 
Nanodrop 2000 (Thermo Scientific) was used to examine the yield and purity of the extracted RNA. Primer sequences were designed by using NCBI database. cDNA synthesis from RNA and qRT-PCR were carried out using SuperScript III Platinum SYBR Green onestep qRT-PCR kit according to the manufacturer's instructions. Reaction conditions for gene expression analyses were as follows: $55^{\circ} \mathrm{C}$ for $5 \mathrm{~min}, 95^{\circ} \mathrm{C}$ for $5 \mathrm{~min}, 40$ cycles of $95^{\circ} \mathrm{C}$ for $15 \mathrm{~s}$, varying temperature depending on the primer sets for $30 \mathrm{~s}$, and $40^{\circ} \mathrm{C}$ for $1 \mathrm{~min}$, followed by a melting curve analysis in order to confirm product specificity. A standard curve was plotted to evaluate the reaction efficiencies for each primer set by using fivefold serial dilutions of total RNA. Target genes used for qRT-PCR were $\beta I I I$ tubulin and synaptophysin as neural differentiation markers and GAPDH as a reference gene. Primary gene expression data was normalized to GAPDH and Ct method (Pfaffl) was used for analyses.

\subsection{Western Blot Analysis}

Total protein samples of PC-12 cells cultured in different conditions (2D or 3D culture, in the presence or absence of NGF) were isolated using TRIzol (Invitrogen) according to the manufacturer's instructions. Protein concentrations were determined using a BCA Protein Assay Kit (Thermo Scientific). Equal amounts of proteins $(50 \mu \mathrm{g}, 15 \mu \mathrm{L})$ were separated on $12 \%$ SDS-PAGE gels under denaturing and nonreducing conditions and then transferred to a PVDF membrane. The membrane was blocked with $5 \%$ nonfat milk in TBSTat room temperature for $1 \mathrm{~h}$ and then incubated with $\beta$ III tubulin antibody (Abcam, ab78078, 1:1000) at $4{ }^{\circ} \mathrm{C}$ overnight. After washing in TBST, the blots were incubated with an HRPconjugated secondary antibody (Millipore, 12-349 goat anti mouse IgG, 1:1000). Signals were visualized using a chemiluminescent signal enhancement system (Invitrogen, Novex ECL). GAPDH was used as the internal control (Millipore, MAB374, 1:500). Gels were visualized by enhanced chemiluminescence (Bio-Rad) according to the manufacturer's protocol on a ChemiDoc ${ }^{\mathrm{TM}}$ Imaging System with Image Lab ${ }^{\mathrm{TM}}$ Software (Bio-Rad), and protein concentrations in gel slabs were quantified by using Image J. Intensities of $\beta$-III tubulin bands were normalized by GAPDH.

\subsection{Statistical Analysis}

All quantitative values are presented as mean \pm SEM (standard error of means), and experiments were performed with at least three replicates. One-way ANOVA was used for statistical analyses, and Bonferroni was used as a post hoc test with ANOVA. All groups were compared with each other, and $p$ values of less than 0.05 were considered statistically significant.

\section{Results}

\subsection{Design and Characterization of Peptide Amphiphile Nanofibers}

In this study, laminin-derived epitope-presenting 3D peptide nanofiber gels were constructed by using LN-PA
(lauryl-VVAGKKIKVAV-Am) to examine the neuronal differentiation potential of PC-12 cells in a 3D environment. Positively charged KK-PA (lauryl-VVAGKK-Am) and negatively charged EE-PA (lauryl-VVAGEE-Am) (Figure 1) were used as nonbioactive molecules for charge neutralization and as epitope-free controls. Peptides were synthesized produced by solid phase peptide synthesis, characterized by LC-MS and purified by HPLC (Figure S1, Supporting Informatiom). Self-assembling PA molecules were designed with a hydrophilic peptide that which is conjugated to a hydrophobic fatty acid, which promotes the self-assembly of PA molecules into nanostructures in aqueous solution. ${ }^{[32]}$ It was also shown that two oppositely charged PAs carrying different bioactive epitopes can self-assemble into nanofibers at physiological conditions due to electrostatic interactions between ionic amino acids of PAs. ${ }^{[33]}$ Noncovalent forces such as hydrogen bonding, hydrophobic and electrostatic interactions between PAs trigger and stabilize the fiber formation. ${ }^{[3]}$ In our study, the KK-PA and LN-PA molecules formed nanofibrous sytems upon mixing with negatively charged EE-PA molecule through these interactions, assembling into a H-bonded $\beta$-sheet secondary structure. ${ }^{[33]}$ Secondary structure analyses of coassembled PAs were performed using circular dichroism (CD) spectroscopy and confirmed that LNPA/EE-PA and KK-PA/EE-PA systems exhibited a $\beta$-sheet conformation, displaying a negative minimum peak around $220 \mathrm{~nm}$ and positive ellipticity around $202 \mathrm{~nm}$ (Figure 2A).

Mechanical properties of LN-PA/EE-PA and KK-PA/EE-PA were analyzed by oscillatory rheology. As the elastic modulus of the ECM has major influence over cellular differentiation patterns, ${ }^{[35]}$ LN-PA/EE-PA and KK-PA/EE-PA gels were prepared to imitate the mechanical properties of the brain ECM, which has an elastic modulus of $\approx 1 \mathrm{kPa}$. Gel components were optimized to the ratios of $(4 \mathrm{mM})$ LN-PA/ $(6 \mathrm{mM})$ EE-PA and $(10 \mathrm{mM}) \mathrm{KK}-\mathrm{PA} /(10 \mathrm{mM})$ EE-PA, as these mixtures exhibited a comparable elastic modulus to brain ECM while retaining an overall neutral charge. According to equilibrium modulus values, storage modulus was greater than the loss modulus for both mixtures, indicating that the resulting gels show elastic solid behavior (Figure 2B, Figure S2 and Table S1, Supporting Information). Both gels were prepared at physiological $\mathrm{pH}$.

Scanning electron microscopy (SEM) was used in order to visualize the 3D nanofiber network of LN-PA/EE-PA and KK-PA/ EE-PA gels. Structural organization of LN-PA/EE-PA and KK-PA/ EE-PA gels was similar to native ECM and could be expected to provide a supportive physical, chemical and biological environment to cells (Figure $2 \mathrm{C}$ and $\mathrm{D}$ ). In addition, zeta potential analysis was performed in order to investigate the charge of the both epitope-free control and laminin-mimetic nanofibers. Charges were found to be around $-10 \mathrm{mV}$, which is in acceptable range of neutral charge (Figure S3, Supporting Information).

\subsection{Neural Differentiation Potential in Laminin Mimetic 2D and 3D Environment}

PC-12 cells were cultured on LN-PA/EE-PA and KK-PA/EE-PA nanofibers (2D) in the presence of NGF to analyze the effect of $2 \mathrm{D}$ culturing on neural differentiation. Neurite length $(\mu \mathrm{m})$ and neurite-bearing cell (\%) analyses were performed using Image J 


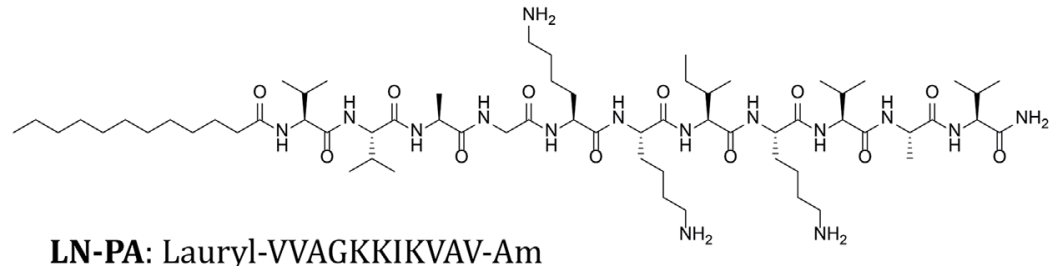
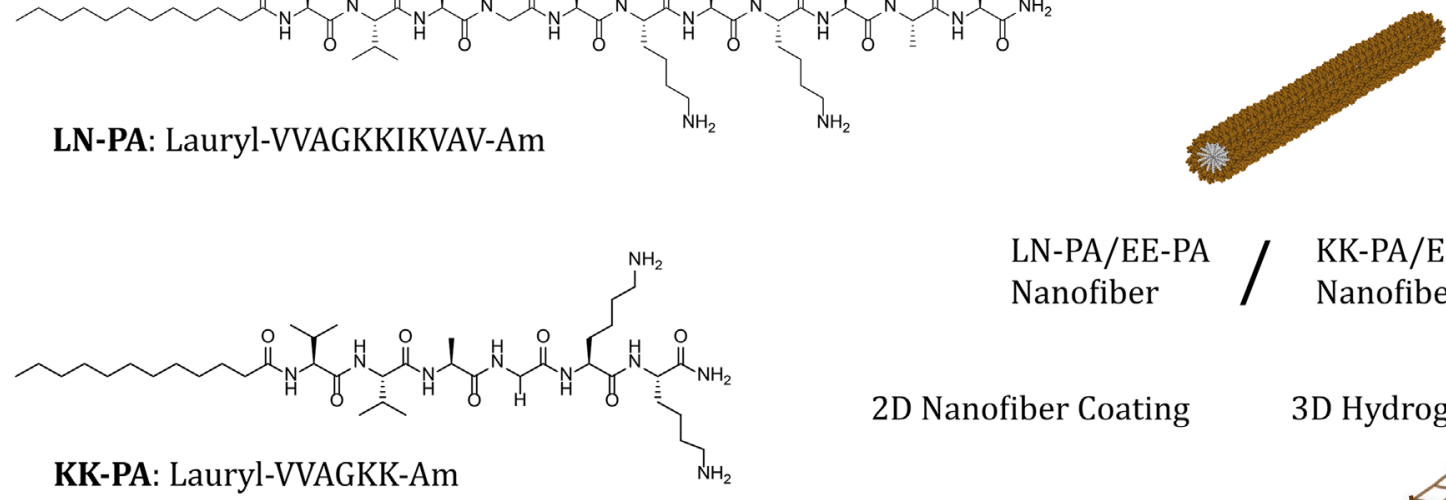

$$
\begin{array}{ll}
\text { LN-PA/EE-PA } \\
\text { Nanofiber }
\end{array} / \begin{aligned}
& \text { KK-PA/EE-PA } \\
& \text { Nanofiber }
\end{aligned}
$$

2D Nanofiber Coating
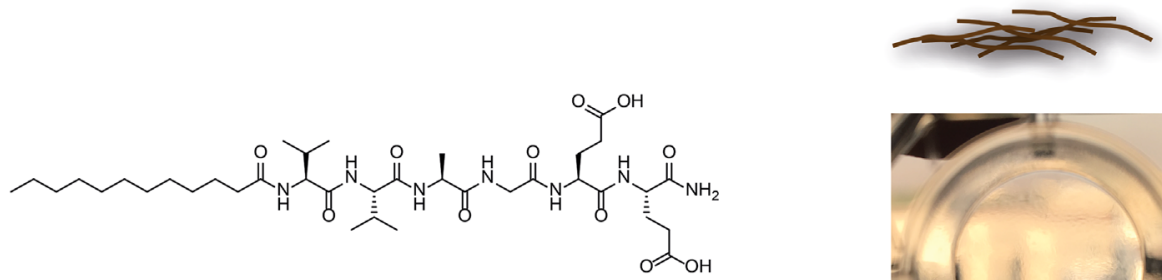

EE-PA: Lauryl-VVAGEE-Am

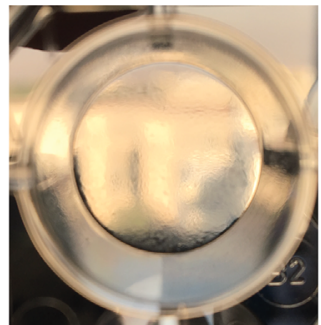

3D Hydrogel Formation
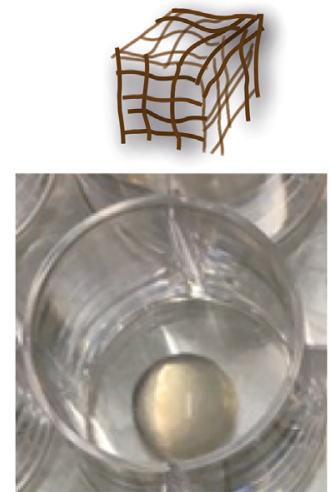

Figure 1. Chemical structures of peptide amphiphile molecules (LN-PA, KK-PA, and EE-PA), and demonstration of 2D nanofiber coating and 3D hydrogels.

A

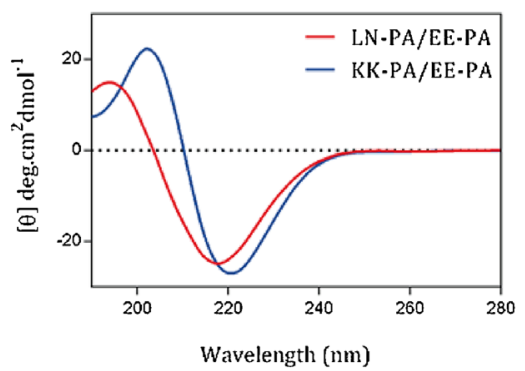

C

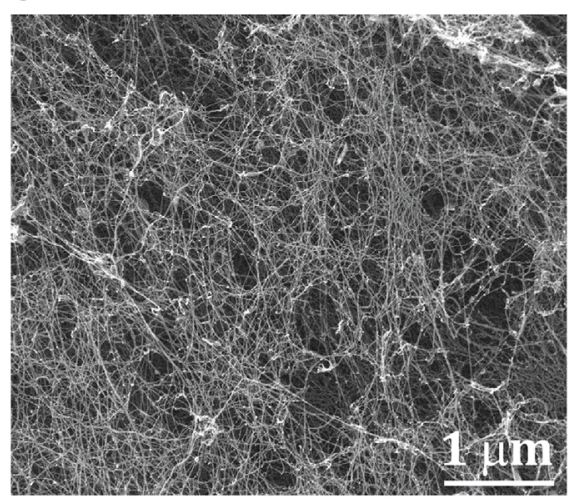

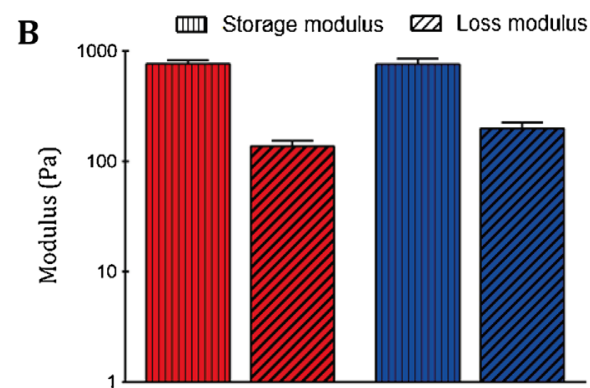

LN-PA/EE-PA

KK-PA/EE-PA

D

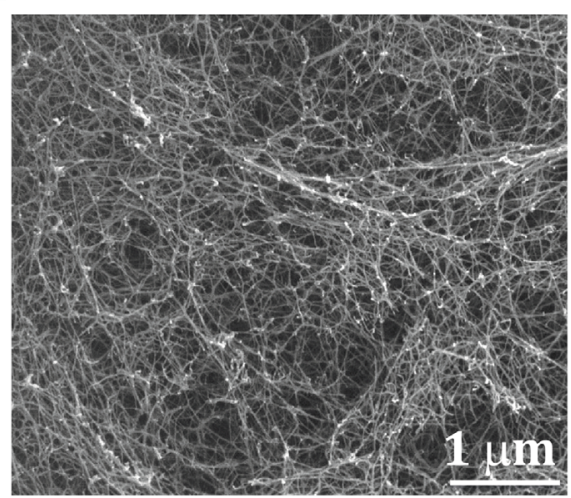

Figure 2. CD spectra of supramolecular LN-PA/EE-PA and KK-PA/EE-PA nanofibers A). Oscillatory rheology measurement showing storage and loss moduli of LN-PA/EE-PA and KK-PA/EE-PA gels in water B). SEM images of LN-PA/EE-PA C) and KK-PA/EE-PA (D) networks. 
LN-PA/EE-PA

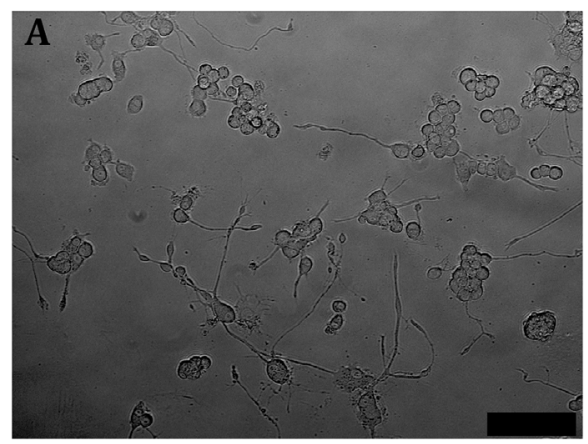

C

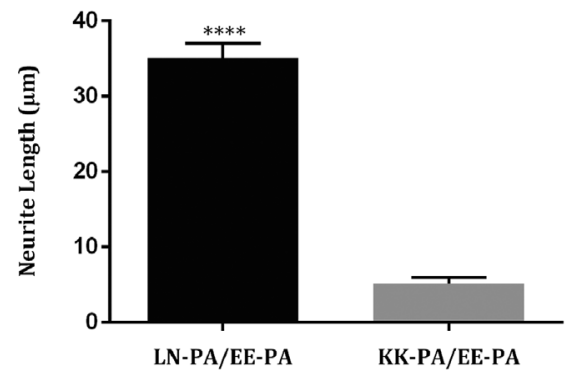

KK-PA/EE-PA
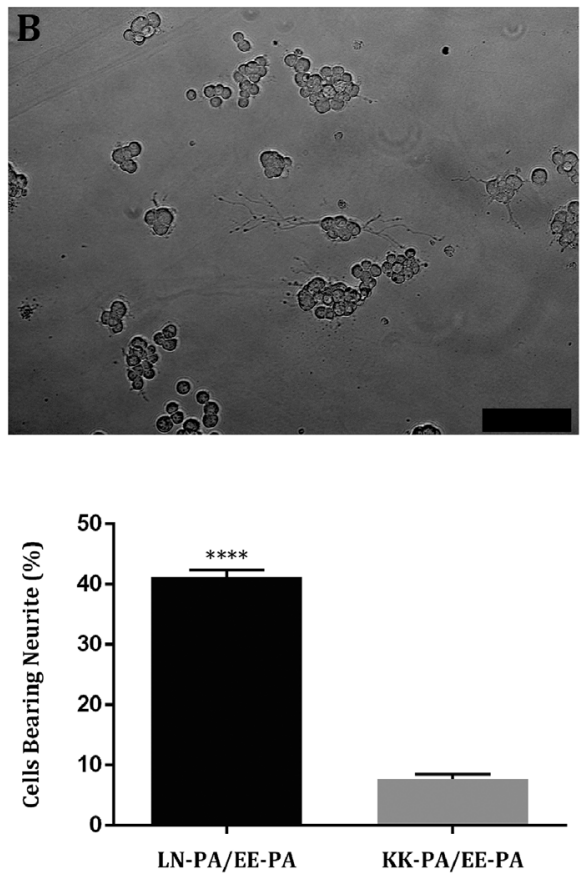

Figure 3. PC- 12 cells cultured on LN-PA/EE-PA A) and KK-PA/EE-PA B) nanofibers (2D) for 7 days with NGF addition, scale bars: $100 \mu m$. Quantification of neurite length and percentage of neurite bearing cells after 7 days $C)$. Values represent mean $\pm \operatorname{SEM}(* * * * p<0.0001)$.

from images taken at the end of the 7-day culture period. The LNPA/EE-PA scaffold significantly enhanced neurite length (Figure 3C) and the number of cells bearing neurites (Figure 3C) compared to the non-bioactive control scaffold. Representative images of cells cultured on LN-PA/EE-PA (Figure 3A) and KK-PA/EE-PA (Figure 3B) indicated that cells exhibited a flat morphology and only bidirectional neuronal outgrowth on 2D culture. Additionally, no neurite outgrowth was observed in either group without NGF addition (Figure S4, Supporting Information).

To further investigate the neural differentiation of PC-12 cells, $\beta$-III tubulin and SYN1 gene expression analyses were carried out by culturing cells on both LN-PA/EE-PA and KK-PA/EE-PA nanofibers (2D) and gels (3D) for 7 days in the presence and absence of NGF. $\beta$-III tubulin is a microtubule element found in the nervous system ${ }^{[36]}$ and synaptophysin (SYN1) is a major synaptic vesicle membrane protein that is expressed in neurons. ${ }^{[37]}$ qRT-PCR analysis showed that the 3D LN-PA/EEPA gel environment significantly enhanced the expression of $\beta$ III tubulin and SYN1 genes, as the cooperative effect of NGF and 3D culturing increased the expression of $\beta$-III tubulin more than twofold and SYN1 more than threefold compared to other groups. The twofold increase in SYN1 expression in 3D (-NGF) scaffolds compared to the 2D (+NGF) group is especially striking and suggests that the regulation of SYN1 is affected by both bioactivity and dimension (Figure 4A). As SYN1 expression was significantly increased in the 3D (-NGF) group compared to both 2D (+NGF) and 2D (-NGF) groups, it is also evident that the bioactive $3 \mathrm{D}$ environment is more effective in eliciting neural differentiation than its 2D counterpart, even when the latter is supplemented by NGF.
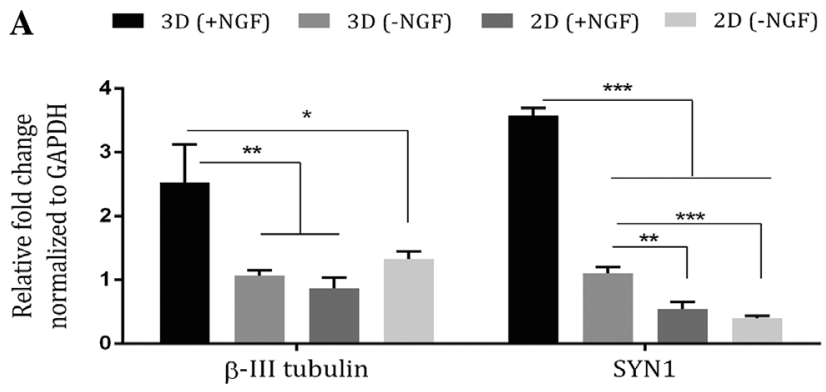

B

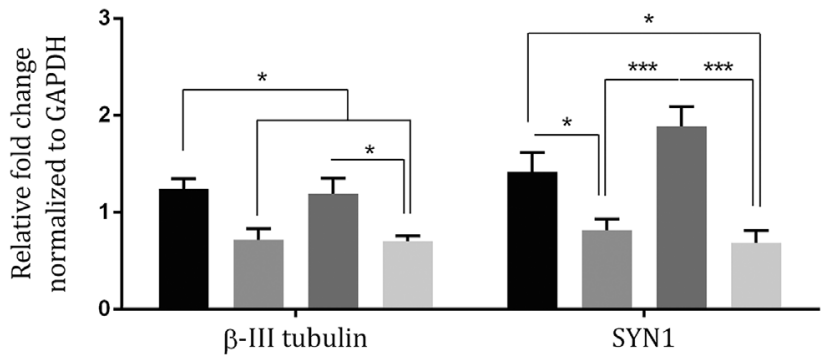

Figure 4. Gene expression analyses of $\beta$-III tubulin and SYN 1 on day 7 on 2D nanofibers and in 3D hydrogels with and without NGF addition. Expression level of each gene was normalized to GAPDH. A) Gene expression levels of PC-12 cells cultured on LN-PA/EE-PA nanofibers and hydrogels. B) Gene expression levels of PC-12 cells on KK-PA/EE-PA nanofibers and hydrogels. Values represent mean $\pm \operatorname{SEM}\left({ }^{* * *} p<0.001\right.$, $\left.{ }^{* *} p<0.01,{ }^{*} p<0.05\right)$. 
In the control groups, expressions of both neural genes were strongly influenced by the presence or absence of NGF, and differences between the groups were not as sharp as under bioactive conditions (Figure 4B). These results demonstrated that, when cells were cultured on nonbioactive scaffolds, NGF presence is more important than scaffold morphology for altering gene expression levels. In contrast, cells cultured on bioactive environments responded both to NGF presence and scaffold morphology, suggesting that a level of interplay occurs between the physical microenvironment and bioactive signaling pathways for the regulation of gene expressions. Additionally, PC-12 cells showed the highest neural differentiation potential in terms of gene expression when cultured in 3D $(+\mathrm{NGF}) \mathrm{LN}$ PA/EE-PA (Figure S6, Supporting Information), further confirming the synergistic effect of bioactivity, dimensionality, and NGF addition.

Neural differentiation of PC-12 cells in 3D scaffolds was further characterized by Western blot analysis to investigate the changes in $\beta$-III tubulin levels at later stages of culture. $\beta$-III tubulin was significantly upregulated at the protein level in 3D LN-PA/EE-PA gels in the presence of NGF (Figure 5). Moreover, cells cultured in 3D KK-PA/EE-PA gels with NGF addition exhibited greater $\beta$-III tubulin expression compared to samples lacking NGF, demonstrating the influence of this growth factor in regulating $\beta$-III tubulin expression when culturing cells in a 3D environment.

Neurite extension and the morphology of PC-12 cells were visualized through SEM imaging after 7 days of culturing on LNPA/EE-PA and KK-PA/EE-PA nanofiber surfaces and gels with and without the addition of NGF. Cells cultured on LN-PA/EEPA nanofiber surfaces and gels showed more neurite extension in the presence of NGF. However, it is notable that neurite extension was also observed without NGF addition in cells cultured in 3D, which underlines the importance of 3D culturing for neural differentiation. Cells cultured in 3D environments also had round morphologies, whereas they had a flatter morphology when cultured in a 2D environment. Different cell morphology and the outgrowth patterns of neurites in different dimensions are the result of cell-to-3D material and cell-to-cell interactions (Figure 6).

\section{Discussion}

Depending on the application of the materials, different types of matrix materials have been used to support 3D cell culture, which includes synthetic polymers, natural polymers, naturalsynthetic hybrids, ceramics, glass, and carbon nanotubes. ${ }^{[38]}$ Within these materials, hydrogel scaffolds, both natural and synthetic hydrogels, are a great option for neural tissue engineering since they can easily be modified to mimic the elastic properties of nervous system. Although synthetic hydrogels are preferred because of their ease to control their physical and chemical properties, they lack the biological activity of natural polymers. Natural hydrogels of macromolecules such as laminin, collagen, and hyaluronic acid have biological activity, however, their use in clinical application is limited due to risk of immune reaction. ${ }^{[39]}$ In contrast, synthetic hydrogels with controlled chemical and physical properties are biodegradable. They can be degraded by cells while the cells synthesize their own ECM. ${ }^{[40]}$ More recent examples of synthetic hydrogels use self-assembling peptide hydrogels which have porous structure similar to natural ECM, and bioactivity can be introduced by incorporating ECM-derived signals. RADA peptides functionalized with IKVAV epitope were used to enhance NSC survival and reduce glial cell formation in 3D system. ${ }^{[41]}$ In another example, neurons encapsulated within aligned nanofibers with TenascinC mimetic signals displayed enhanced neurite outgrowth, and also these nanofibers were found to promote neurospherederived cell migration. ${ }^{[16]}$

The present study emphasizes the differences between 2D and 3D cell culturing for neural differentiation. Neural differentiation potential of $\mathrm{PC} 12$ cells was investigated on 2D and in 3D peptide nanofiber scaffolds presenting laminin mimetic bioactive signal or control sequence, with or without the addition of external NGF. Compared to controls, the laminin epitope IKVAV could better stimulate neurite outgrowth and neurogenic gene expression when cells were cultured on 2D surfaces, suggesting that bioactive signals have a greater impact on neural differentiation under 2D culture environments. Despite offering more modest improvements

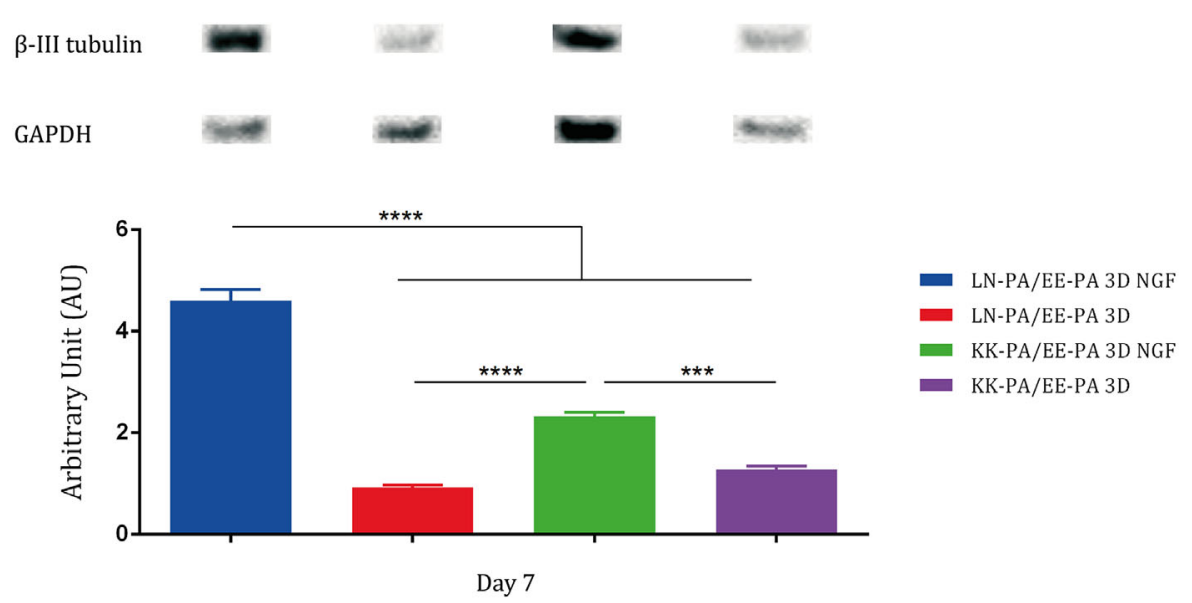

Figure 5. $\beta$-III tubulin expression of cells cultured in 3D hydrogels with and without NGF addition on day 7 . The density of the bands were analyzed by Image ) and normalized to GAPDH. Values represent mean \pm SEM $\left({ }^{* * * *} p<0.0001,{ }^{* * *} p<0.001\right)$. 

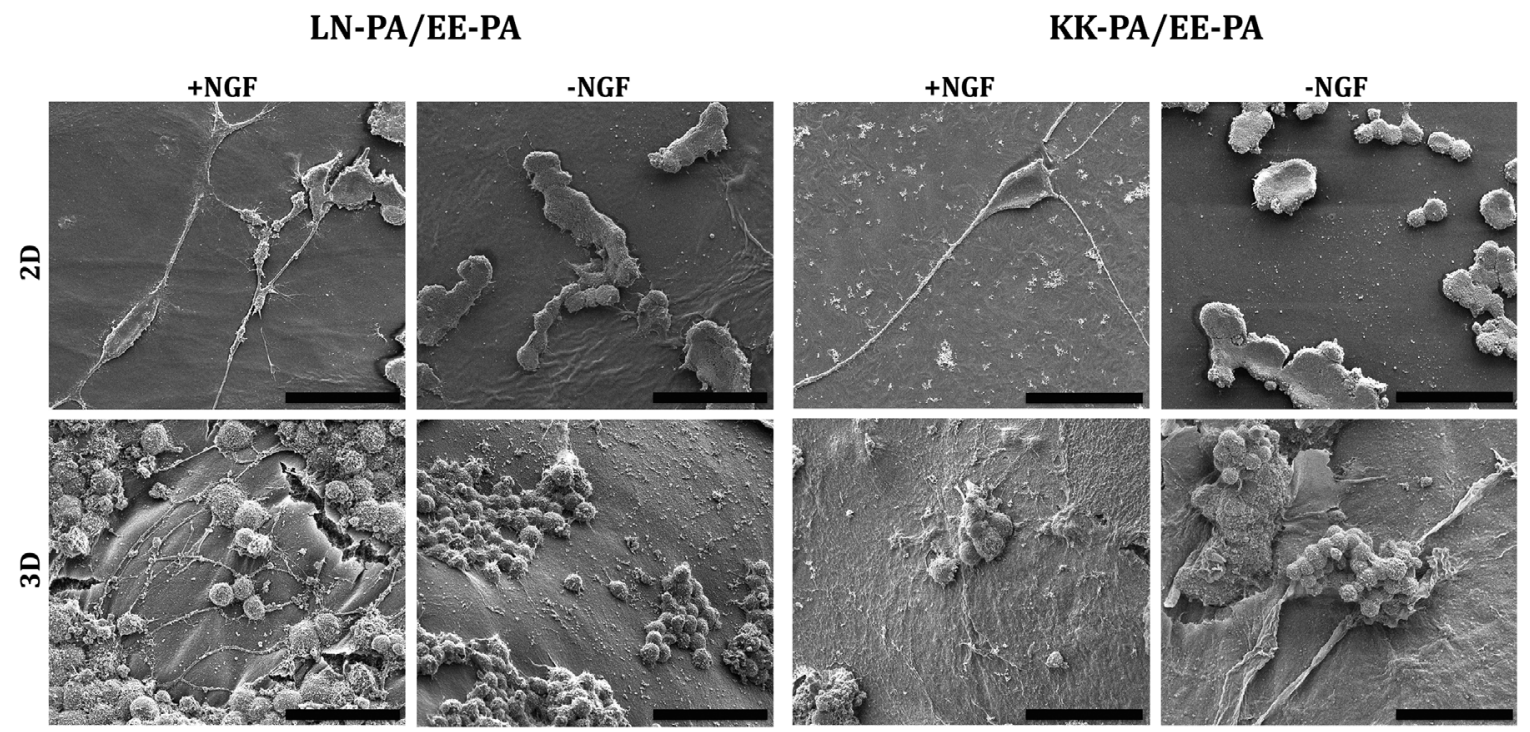

Figure 6. Neurite extension and morphological characteristics of PC-12 cells cultured on 2D and 3D LN-PA/EE-PA and KK-PA/EE-PA in the presence and absence of NGF. Scale bar is $40 \mu \mathrm{m}$.

over controls, the IKVAV epitope also had synergistic effects with 3D culture conditions, as the combination of the bioactive gel and 3D environment stimulated neurogenesis to a greater extent than all other groups. Strikingly, synaptophysin gene expression was significantly higher in the NGF- 3D bioactive group compared to the NGF+ 2D bioactive group, indicating the effect of dimensionality on gene expression. Additionally, Western blot analysis indicated that cells cultured in bioactive 3D scaffolds show significant upregulation in $\beta$-III tubulin protein expression, and neurite extension was also enhanced when cells are cultured in a bioactive laminin-mimetic 3D microenvironment. This distinction is vital for mimicking the native extracellular environment of neural systems, as mammalian brains permit cell-cell and cell-ECM interactions in all three dimensions - indeed cells were only observed to extend their neurites in two dimensions on 2D surfaces, while they could extend their neurites in three dimensions when cultured in 3D scaffolds.

Overall, the neural differentiation potential of PC- 12 cells was investigated on 2D and in 3D peptide nanofiber systems presenting laminin-mimetic bioactive signals with or without exogenous NGF addition, and scaffold morphology was found to be crucial in determining the extent of differentiation and responsiveness to NGF. It is essential to understand and replicate cellular behavior in in vitro conditions for developing better in vivo applications; and the 3D scaffold described in the present study accomplishes this by presenting a threedimensional environment similar to that found in native ECM, mimicking the mechanical characteristics of brain tissue, and incorporating a laminin mimetic IKVAV bioactive epitope for a close representation of in vivo conditions. Overall, it is possible to combine biological signals and mechanical properties into peptide nanofiber scaffolds and further improve their usage for regeneration under neurodegenerative disease conditions.

\section{Supporting Information}

Supporting Information is available from the Wiley Online Library or from the author.

\section{Acknowledgements}

G. G. and M. S. contributed equally to this work. We thank Z. Erdogan and M. G. for their technical help in purification and characterization of PA nanofibers. M.S. is supported by TUBITAK-BIDEB (2211) Ph.D. fellowship, and G.G. is supported by TUBITAK-BIDEB (2210-C) M.Sc. fellowship. M.O.G. and A.B.T. acknowledge support from the Turkish Academy of Sciences Distinguished Young Scientist Award (TUBAGEBIP).

\section{Conflict of Interest}

The authors declare no conflicts of interest.

\section{Keywords}

2D and 3D cell culture, extracellular matrix, laminin, neural differentiation, peptide amphiphile

Received: March 21, 2017

Revised: June 26, 2017

Published online: September 22, 2017

[1] A. Khoruzhenko, Biopolym. Cell 2011, 27, 17.

[2] M. Yu, S. Huang, K. J. Yu, A. M. Clyne, Int. J. Mol. Sci. 2012, 13, 5554.

[3] T. Sun, S. Jackson, J. W. Haycock, S. MacNeil, J. Biotechnol. 2006, 122, 372.

[4] K. Chitcholtan, E. Asselin, S. Parent, P. H. Sykes, J. J. Evans, Exp. Cell Res. 2013, 319, 75. 
[5] M. Pickl, C. Ries, Oncogene 2009, 28, 461.

[6] X. F. Tian, B. C. Heng, Z. Ge, K. Lu, A. J. Rufaihah, V. T. Fan, J. F. Yeo, T. Cao, Scand. J. Clin. Lab. Invest. 2008, 68, 58.

[7] H. Baharvand, S. M. Hashemi, S. K. Ashtiani, A. Farrokhi, Int. J. Dev. Biol. 2004, 50, 645 .

[8] K. Y. Lee, D. J. Mooney, Progr. Polym. Sci. 2012, 37, 106.

[9] V. V. Artym, K. Matsumoto, Curr. Protoc. Cell Biol. 2010, 48, 10.18:10.18.1.

[10] H. K. Kleinman, M. L. McGarvey, L. A. Liotta, P. G. Robey, K. Tryggvason, R. G. Martin, Biochemistry 1982, 21, 6188.

[11] N. C. Hunt, D. Hallam, A. Karimi, C. B. Mellough, C. Chen, D. H W. Steel, M. Lako, Acta Biomater. 2016.

[12] M.-N. Labour, A. Banc, A. Tourrette, F. Cunin, J. M. Verdier, J. M. Devoisselle, A. Marcilhac, E. Belamie, Acta Biomater. 2012, 8, 3302.

[13] E. Arslan, M. O. Guler, A. B. Tekinay, Biomacromolecules 2016, 17, 1280.

[14] Y. Loo, M. Goktas, A. B. Tekinay, M. O. Guler, C. A. Hauser, A. Mitraki, Adv. Healthc. Mater. 2015, 4, 2557.

[15] M. S. Ekiz, G. Cinar, M. A. Khalily, M. O. Guler, Nanotechnology 2016, 27, 402002

[16] E. J. Berns, Z. Álvarez, J. E. Goldberger, J. Boekhoven, J. A. Kessler, H. G. Kuhn, S. I. Stupp, Acta Biomater. 2016, 37, 50.

[17] B. Mammadov, R. Mammadov, M. O. Guler, A. B. Tekinay, Acto Biomater. 2012, 8, 2077.

[18] M. D. Pierschbacher, E. Ruoslahti, Nature 1984, 309, 30.

[19] J. Graf, R. C. Ogle, F. A. Robey, M. Sasaki, G. R. Martin, Y. Yamada, H. K. Kleinman, Biochemistry 1987, 26, 6896.

[20] K. R. Legate, S. A. Wickström, R. Fässler, Genes Dev. 2009, 23, 397.

[21] R. O. Hynes, Cell 2002, 110, 673.

[22] V. Marthiens, I. Kazanis, L. Moss, K. Long, J. Cell. Sci. 2010, 123, 1613.

[23] L. S. Campos, L. Decker, V. Taylor, W. Skarnes, J. Biol. Chem. 2006, 281,5300

[24] N. J. Gardiner, Dev. Neurobiol. 2011, 71, 1054.
[25] X. Li, X. Liu, B. Josey, C. J. Chou, Y. Tan, N. Zhang, X. Wen, Stem Cells Transl. Med. 2014, 3, 662.

[26] K.-i. Tashiro, G. C. Sephel, B. Weeks, M. Sasaki, G. R. Martin, H. K. Kleinman, Y. Yamada, J. Biol. Chem. 1989, 264, 16174.

[27] G. A. Silva, C. Czeisler, K. L. Niece, E. Beniash, D. A. Harrington, J. A. Kessler, S. I. Stupp, Science 2004, 303, 1352.

[28] V. M. Tysseling-Mattiace, V. Sahni, K. L. Niece, D. Birch, C. Czeisler, M. G. Fehlings, S. I. Stupp, J. A. Kessler, J. Neurosci. 2008, 28, 3814.

[29] P. C. Georges, W. J. Miller, D. F. Meaney, E. S. Sawyer, P. A. Janmey, Biophys. J. 2006, 90, 3012.

[30] K. Saha, A. J. Keung, E. F. Irwin, Y. Li, L. Little, D. V. Schaffer K. E. Healy, Biophys. J. 2008, 95, 4426.

[31] M. Sever, B. Mammadov, M. Gecer, M. O. Guler, A. B. Tekinay, Ther. Nanomater. 2016, 33.

[32] J. D. Hartgerink, E. Beniash, S. I. Stupp, Proc. Natl. Acad. Sci. 2002 99, 5133

[33] K. L. Niece, J. D. Hartgerink, J. J. Donners, S. I. Stupp, J. Am. Chem Soc. 2003, 125, 7146.

[34] S. E. Paramonov, H.-W. Jun, J. D. Hartgerink, J. Am. Chem. Soc. 2006 128, 7291.

[35] A. Engler, H. Sweeney, D. Discher, J. Schwarzbauer, J. Musculoskel. Neuronal Interact. 2007, 7, 335.

[36] E. Dráberová, L. Del Valle, J. Gordon, V. Marková, B. Smejkalová L. Bertrand, J. P. de Chadarévian, D. P. Agamanolis, A. Legido, K. Khalili, P. Dráber, C. D. Katsetos, J. Neuropathol. Exp. Neurol. 2008 67, 341.

[37] B. Marqueze-Pouey, W. Wisden, M. Malosio, H. Betz, J. Neurosci. 1991, 11, 3388

[38] A. R. Murphy, A. Laslett, C. M. O'Brien, N. R. Cameron, Acta Biomater. 2017, 54, 1.

[39] Y. Shao, J. Sang, J. Fu, Biomaterials 2015, 52, 26.

[40] J. Zhu, R. E. Marchant, Expert Rev. Med. Devices 2011, 8, 607.

[41] T.-Y. Cheng, M.-H. Chen, W.-H. Chang, M.-Y. Huang, T.-W. Wang, Biomaterials 2013, 34, 2005. 